\title{
Surgically implanted aortic valve bioprostheses deform after implantation: insights from computed tomography
}

\author{
Marguerite E. Faure ${ }^{1,2}$ (D) Dominika Suchá ${ }^{3} \cdot$ Fides R. Schwartz ${ }^{4} \cdot$ Petr Symersky $^{5} \cdot$ Ad J. J. C. Bogers $^{6}$. \\ Jeffrey G. Gaca ${ }^{7}$ - Lynne M. Koweek ${ }^{4}$. Linda M. de Heer ${ }^{8,9} \cdot$ Ricardo P. J. Budde $^{1}$
}

Received: 6 August 2019 / Revised: 28 November 2019 / Accepted: 13 December 2019

(C) European Society of Radiology 2020

\begin{abstract}
Objective Little is known about the prevalence and degree of deformation of surgically implanted aortic biological valve prostheses (bio-sAVRs). We assessed bio-sAVR deformation using multidetector-row computed tomography (MDCT).

Methods Three imaging databases were searched for patients with MDCT performed after bio-sAVR implantation. Minimal and maximal valve ring diameters were obtained in systole and/or diastole, depending on the acquired cardiac phase(s). The eccentricity index (EI) was calculated as a measure of deformation as $(1-($ minimal diameter/maximal diameter $)) \times 100 \%$. EI of $<5 \%$ was considered none or trivial deformation, 5-10\% mild deformation, and $>10 \%$ non-circular. Indications for MDCT and implanted valve type were retrieved.

Results One hundred fifty-two scans of bio-sAVRs were included. One hundred seventeen measurements were performed in systole and 35 in diastole. None or trivial deformation $(\mathrm{EI}<5 \%)$ was seen in 67/152 (44\%) of patients. Mild deformation (EI 5-10\%) was seen in 59/152 (39\%) and non-circularity was found in 26/152 (17\%) of cases. Overall, median EI was 5.5\% (IQR 3.4-7.8). In 77 patients, both systolic and diastolic measurements were performed from the same scan. For these scans, the median EI was $6.5 \%$ (IQR 3.4-10.2) in systole and 5.1\% (IQR3.1-7.6) in diastole, with a significant difference between both groups ( $p=0.006$ ).

Conclusions Surgically implanted aortic biological valve prostheses show mild deformation in $39 \%$ of cases and were considered non-circular in $17 \%$ of studied valves.

Key Points

- Deformation of surgically implanted aortic valve bioprostheses (bio-sAVRs) can be adequately assessed using MDCT.

- Bio-sAVRs show at least mild deformation (eccentricity index $>5 \%$ ) in 56\% of studied cases and were considered non-circular (eccentricity index $>10 \%$ ) in $17 \%$ of studied valves.

- The higher deformity rate found in bio-sAVRs with (suspected) valve pathology could suggest that geometric deformity may play a role in leaflet malformation and thrombus formation similar to that of transcatheter heart valves.
\end{abstract}

Keywords Heart valve prosthesis · Bioprosthesis · Multidetector-row computed tomography

Marguerite E. Faure and Dominika Suchá contributed equally to this work.

Electronic supplementary material The online version of this article (https://doi.org/10.1007/s00330-019-06634-6) contains supplementary material, which is available to authorized users.

Marguerite E. Faure

m.faure@erasmusmc.nl

1 Department of Radiology and Nuclear Medicine, Erasmus Medical Center, Po Box 2040, 3000, CA Rotterdam, The Netherlands

2 Department of Radiology, AZ Monica, Antwerp, Belgium

3 Department of Radiology, University Medical Center Utrecht, Utrecht, The Netherlands

4 Department of Radiology, Duke University Medical Center, Durham, USA
5 Department of Cardiothoracic Surgery, VU Medical Center, Amsterdam, The Netherlands

6 Department of Cardiothoracic Surgery, Erasmus Medical Center, Rotterdam, The Netherlands

7 Department of Cardiothoracic Surgery, Duke University Medical Center, Durham, USA

8 Department of Cardiothoracic Surgery, Leiden University Medical Center, Leiden, The Netherlands

9 Department of Cardiothoracic Surgery, University Medical Center Utrecht, Utrecht, The Netherlands 


\begin{tabular}{ll}
\multicolumn{2}{l}{ Abbreviations } \\
AVR & Aortic valve replacement \\
Bio-sAVR & Biological surgical aortic valve replacement \\
EI & Eccentricity index \\
IQR & Interquartile range \\
MDCT & Multidetector-row computed tomography \\
SAVR & Surgical aortic valve replacement \\
SVD & Structural valve deterioration \\
TAVI & Transcatheter aortic valve implantation \\
THV & Transcatheter heart valve
\end{tabular}

\section{Introduction}

Approximately 280,000 valve prostheses are implanted worldwide each year: $20 \%$ of which are mechanical valves and $80 \%$ bioprosthetic valves. Bioprostheses are increasingly chosen due to favorable hemodynamic profile, longer durability with recent generations of bioprosthetic valves, and low thrombogenicity [1, 2]. In spite of significant improvement of bioprosthesis design and surgical procedures, the implantation does not necessarily result in a definitive cure, and native valve disease is often replaced by "prosthetic valve disease" [3]. Structural valve deterioration (SVD) is the major cause of surgical aortic valve bioprosthesis (bio-sAVR) failure, which increases with time post implantation. The reoperation rate for biological valves in the aortic position is $10 \%$ at 10 years and $30 \%$ at 15 years [4]. In transcatheter heart valves (THV), deformity of the valves after implantation has been a key concern in several studies [5-8] and non-circularity of the prosthesis has been found in up to a third of cases [9-11]. The self-expandable valves in particular show deformation of the valve in up to $36 \%$ of cases at the level of the aortic annulus $[10,12,13]$. Deformation of surgical biological valves may result in incomplete leaflet expansion, and subsequently accelerate valve degeneration; it may also alter mechanical stress distribution and facilitate valve leaflet thrombosis and calcification [14-16] but has not been assessed in large prospective clinical studies. Most bio-sAVRs have a frame that supports the leaflets but this frame can be easily deformed by external forces (Video File 1). It is conceivable that deformation of bio-sAVR after implantation could occur post implantation. The aim of this study was to assess the deformity of bio-sAVR after surgical implantation using multidetector-row computed tomography (MDCT).

\section{Patients and methods}

\section{Patients}

We performed a retrospective search in three medical databases (UMC Utrecht 2007-2014, EMC Rotterdam 2012-
2019, and Duke UMC Durham 2016-2018), for patients with a bio-sAVR and available ECG-gated MDCT exams. Stentless valves and THVs were excluded. In patients with multiple ECG-gated MDCT acquisitions, the acquisition closest to date of implantation was included for analysis. The implanted valve type and size, indication for MDCT, and time from implantation to MDCT imaging were collected. Both prospectively ECG-gated and retrospectively ECG-gated acquisitions were included.

\section{Image analysis}

For each acquisition, we selected the best systolic and diastolic cardiac phase with least bio-sAVR-related artifacts and best subjective image quality. When both systolic and diastolic phases were available, systolic measurements were utilized for overall measurements. We preferred systolic measurements because this is considered the most important phase for sizing in pre-surgical imaging.

Images were reconstructed in three planes using the multiplanar tool available on a dedicated commercially available workstation (Intellispace Portal, Philips Healthcare, and Syngo.via, Siemens Healthineers). Images were reconstructed exactly in plane enface with the prosthesis. Three observers, DS, MEF, and FRS, with respectively 7-, 5-, and 1-year experience in cardiac imaging, measured the maximal and minimal inner valve diameter for each valve in systole and diastole, when available. This was done by tracing the perimeter at the level of the bio-sAVR ring and/or stent where it was visible as a complete (circular) structure. The software automatically generated minimal and maximal diameter measurements perpendicular to each other, as presented in Fig. 1 and Video File 2.

The eccentricity index (EI) was calculated for both cardiac phases as $\mathrm{EI}=(1-($ minimal diameter $/$ maximal diameter $)) \times$ $100 \%$. The eccentricity of the bio-sAVR was considered none/ trivial (circular) with an EI $<5 \%$, mild with an EI 5-10\%, and non-circular with an EI of $>10 \%$ (Fig. 2) [7, 9].

\section{Statistical analysis}

IBM SPSS Statistics 24.0 software was used for analysis. QQplots and Shapiro-Wilk test were performed to evaluate data distribution. Categorical data are presented as number and percentage, continuous data as mean \pm standard deviation, or median and interquartile range (IQR) as appropriate. Since data were not normally distributed, the Wilcoxon signed rank test was used for paired systolic and diastolic EI measurement comparison. For independent data, the Mann-Whitney $U$ test was used. A $p$ value of $\leq 0.05$ was considered significant. 

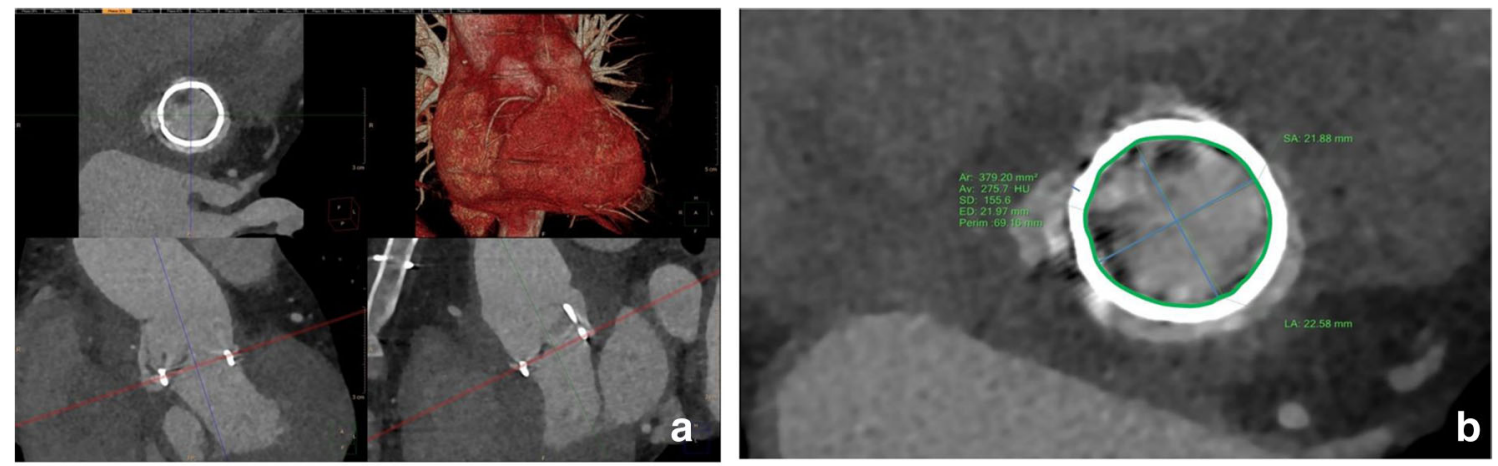

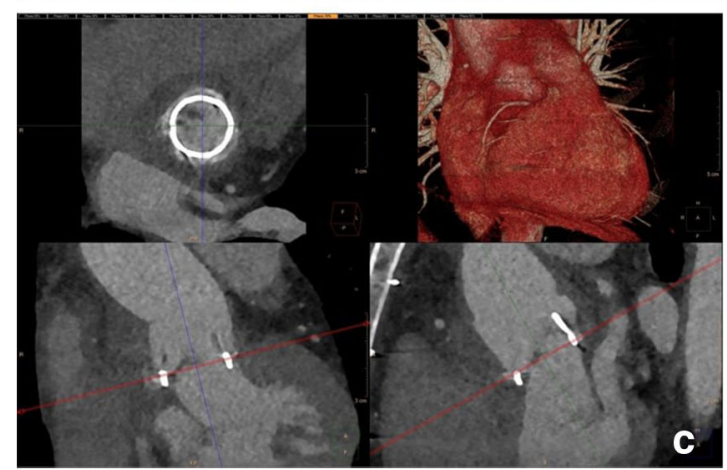

Fig. 1 Demonstration of measurement acquisition: example of in-plane measurements of the minimal and maximal inner valve diameters of a biological valve prosthesis in aortic position. In this patient, both systolic (a, b) and diastolic (c, d) measurements were available. Images were reviewed in three planes using a multiplanar tool, reconstructing an

\section{Results}

Two hundred patients with stented biological valves in the aortic position and ECG-gated MDCT imaging exams were identified. Of these, 34 were transcatheter valves and excluded from further analysis. Twelve Mitroflow aortic valves (LivaNova PLC) were excluded due to the unique structure with leaflets mounted outside the stent frame and a radiopaque sewing ring that is always eccentric as it adapts to the annulus. Two patients were excluded due to notable artifacts rendering accurate measurements unobtainable. The final cohort

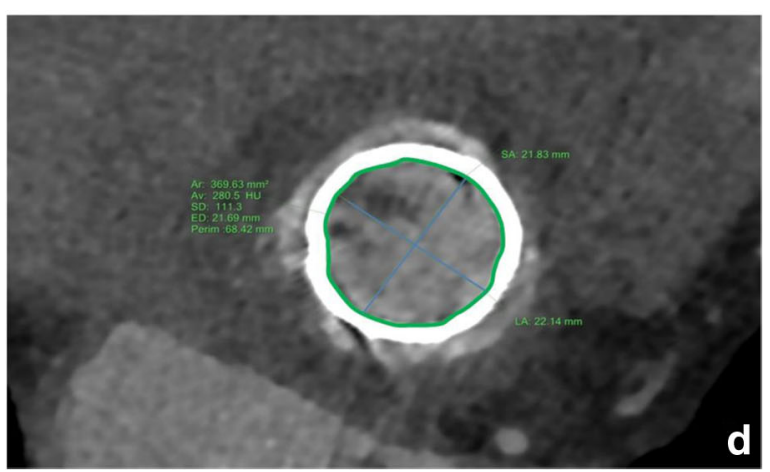

image exactly in plane enface with the prosthesis $(\mathbf{a}, \mathbf{c})$. Perimeter was traced at the level of the AVR ring and/or stent where it was visible as a complete (circular) structure. The software automatically generated minimal and maximal diameters perpendicular to each other $(\mathbf{b}, \mathbf{d})$

included 152 scans of bio-sAVR (Table 1). The vast majority of studied valves concerned the Carpentier-Edwards Perimount valve (Edwards Lifesciences Inc.): 120/152 valves (79\%).

In 84 of 152 scans (55\%), bio-sAVR MDCT was performed for routine postoperative follow-up, study purposes, or for other reasons than primary prosthetic valve assessment. In 41 of 152 scans (27\%), MDCT was performed for evaluation of suspected bio-sAVR endocarditis, regurgitation, obstruction, or dysfunction. In the remaining 27 of 152 scans (18\%), MDCT performed as part of pre-surgical planning,

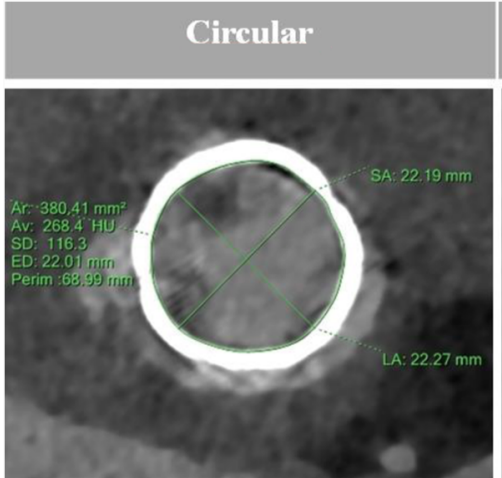

Fig. 2 Range of eccentricity of biologic surgical aortic valves on cardiac MDCT exams: examples of varying degree of eccentricity of the same type of aortic biological valve in three different patients. Eccentricity was defined as follows: none/trivial (circular) $<5 \%$, mild 5-10\%, and noncircular $>10 \%$ eccentricity based on the eccentricity index calculated as $(1-($ minimal diameter/maximal diameter $)) \times 100$ 
e.g., pre "transcatheter aortic valve implantation" (TAVI) in failed bio-sAVR. Median time between bioprosthesis implantation and MDCT was 12 months (IQR 2-82). For one patient, implantation date was unknown. Overall, 52/151 (34\%) scans were performed less than 3 months after implantation, 23/151 (23\%) scans between 3 and 12 months after implantation, and $76 / 151(50.5 \%)$ scans more than 12 months after implantation. In this last group, 33/151 (22\%) were performed between 1 and 5 years after implantation and 43/151 (28.5\%) were acquired more than 5 years after.

Of the 152 scans, 117 had systolic measurements and 112 had diastolic measurements, resulting in a total of 229 measurements performed for the 152 scans. In these 152 scans, 77 had systolic and diastolic measurements since both phases

Table 1 Parameters of surgical aortic biological valves assessed

Total, $N=152$

\begin{tabular}{lr} 
Bioprosthesis type & \\
Carpentier-Edwards Perimount (Edwards Lifesciences) & $120(79 \%)$ \\
Mosaic (Medtronic) & $7(4.6 \%)$ \\
CE Perimount Magna Aortic (Edwards Lifesciences) & $11(7.2 \%)$ \\
Biocor/Epic (St. Jude Medical*) & $2(1.3 \%)$ \\
Avalus (Medtronic) & $1(0.7 \%)$ \\
Edwards Intuity Elite (Edwards Lifesciences) & $1(0.7 \%)$ \\
Mosaic Ultra (Medtronic) & $1(0.7 \%)$ \\
Trifecta (St Jude Medical*) & $1(0.7 \%)$ \\
Perceval (Sorin Group**) & $1(0.7 \%)$ \\
Unknown & $7(4.6 \%)$ \\
Prosthesis size & \\
19 mm & $2(1.3 \%)$ \\
21 mm & $19(12.5 \%)$ \\
23 mm & $39(25.7 \%)$ \\
25 mm & $51(33.6 \%)$ \\
27 mm & $31(20.4 \%)$ \\
29 mm & $2(1.3 \%)$ \\
Unknown & $8(5.3 \%)$ \\
Reason for performing MDCT & \\
Postoperative evaluation (no pathology) & $33(21.7 \%)$ \\
Research purpose (no pathology) & $32(21.1 \%)$ \\
Other - NonPHV-related & $19(12.5 \%)$ \\
Presurgery/TAVI (failed AVR) & $27(17.7 \%)$ \\
Suspected endocarditis & $22(14.5 \%)$ \\
Suspected obstruction & $7(4.6 \%)$ \\
Suspected leakage (non-endocarditis) & $3(2.0 \%)$ \\
\hline & $9(5.9 \%)$ \\
& $12[2-82]$ \\
\hline
\end{tabular}

Data presented as number (percentage) or median [interquartile range]

*Acquired by Abbott Laboratories

**Merged in LivaNova PLC were scanned in these patients. Forty scans had systolic measurements only and 35 scans had diastolic measurements only. If both systolic and diastolic measurements were available, systolic measurements were utilized for the analysis. Therefore, a total of 117 systolic measurements and 35 diastolic measurements were used for the overall analysis. None or trivial deformation $(\mathrm{EI}<5 \%)$ was seen in $67 / 152(44 \%)$ of patients, 49/117 (42\%) in systole, and 18/35 (51\%) in diastole. Mild deformation (EI 5-10\%) was seen in 59/152 (39\%) scans overall and 45/117 (38\%) and 14/35 (40\%) measurements in systole and diastole, respectively; whereas non-circularity was found in a total of 26/152 (17\%) scans, 23/128 (20\%) and 3/35 $(9 \%)$ in systole and diastole, respectively (Table 2). Only one of these non-circular valves had an EI $>20 \%$, with an EI of 30.8 in systole and 26.7 in diastole. Overall, median EI was $5.5 \%$ (IQR 3.4-7.8) for all 152 scans, 5.8\% (IQR 3.4-8.5) in the 117 systolic scans, and 4.9\% (IQR 2.7-6.6) in the 35 diastolic scans, which was significantly different $(p=0.05)$.

In 77 patients, measurements in both systole and diastole were performed. For these scans, the median EI was 6.5\% (IQR 3.4-10.2) in systole and 5.1\% (IQR3.1-7.6) in diastole, with a significant difference between both groups $(p=0.006)$.

Table 3 shows median valve eccentricity, stratified by valve type. There was a significant difference between the median EI of scans performed for suspected valve pathology and scans done for follow-up, study purpose, or other pathologies. In the group with suspected valve pathology, median EI was 6.9\% (IQR3.4-10.6) compared with median EI 5.0\% (IQR3.3-6.9) in the group without suspected dysfunction $(p=0.023)$.

\section{Discussion}

In the current study, we evaluated the degree of geometrical deformation of aortic biological valve prostheses after surgical implantation on MDCT and found a considerable number of bioprostheses to show valve deformation. At least mild deformation was found in 56\% (85/152) of biological valves of which $17 \%$ (26/152) were considered non-circular.

Table 2 Degree of deformation of biological surgical aortic valve

\begin{tabular}{llll}
\hline & $\begin{array}{l}\text { Total } \\
N=152\end{array}$ & $\begin{array}{l}\text { Systolic phase } \\
N=117\end{array}$ & $\begin{array}{l}\text { Diastolic phase } \\
N=35\end{array}$ \\
\hline None/trivial eccentricity & $67(44 \%)$ & $49(42 \%)$ & $18(51 \%)$ \\
Mild eccentricity & $59(39 \%)$ & $45(38 \%)$ & $14(40 \%)$ \\
Non-circular & $26(17 \%)$ & $23(20 \%)$ & $3(9 \%)$
\end{tabular}

Eccentricity was defined as follows: none/trivial $<5 \%$, mild $5-10 \%$, and non-circular $>10 \%$ eccentricity 
Table 3 Degree of eccentricity of biological surgical aortic valve degree related to time of reconstruction in the cardiac cycle

\begin{tabular}{lrrrrc}
\hline & \multicolumn{2}{l}{ Eccentricity* } & & \\
\cline { 2 - 5 } Biological valve type & $N$ & \multicolumn{1}{l}{ Systolic } & $N$ & Diastolic & $p$ value \\
\hline CE Perimount & 92 & $5.2 \%[3.1-7.4 \%]$ & 84 & $4.7 \%[2.8-6.3 \%]$ & 0.21 \\
Medtronic Mosaic & 6 & $10.5 \%[7.0-14.0]$ & 7 & $10.6 \%[4.0-11.3]$ & 0.39 \\
CE Perimount Magna aortic & 8 & $7.7 \%[6.0-10.7]$ & 10 & $4.9 \%[3.1-9.2]$ & 0.11 \\
Other types/unknown & 11 & $6.8 \%[3.4-10.6 \%]$ & 11 & $5.5 \%[2.9-7.8 \%]$ & 0.31 \\
\hline
\end{tabular}

$N=$ total number valves evaluated; since some valves are measured in both systole and diastole, total number of valves in this table exceeds 160; *Presented as median [interquartile range], measured as (1- (minimal diameter/ maximal diameter) $) \times 100$
Previous studies have shown that mechanical stress can impact the (pathological) tissue calcification process, which affects valve durability $[3,14,15,17,18]$. It has also been suggested that structural deformation might contribute to the valve calcification process $[14,19]$. As deformation of the prosthetic heart valve stent can impact leaflet composition and stress distribution, bioprostheses with significant deformation may be more prone to leaflet thrombosis and (early) structural valve deterioration [16]. Our study found a significant higher deformity in bio-sAVRs with (suspected) valve pathology as compared with supposedly normal functioning valves, supporting this hypothesis.

We found a considerable portion of implanted bio-sAVRs to show deformation of any extent. Literature on the prevalence and degree of deformation of surgical bioprostheses is, however, scarce. To our knowledge, this is the first study assessing the geometry of surgically implanted biological aortic valves on CT. Deformation of THVs has been evaluated extensively, showing non-circularity in $0-34 \%$ balloonexpandable and up to $87 \%$ in self-expandable valves [5-7, $9,11,20-22]$. These results can, however, not be extrapolated to surgical valves, since surgical valves differ fundamentally from THVs both in configuration and structure as well as technique of implantation. Hence, the underlying mechanism of deformation is likely to differ between bio-sAVRs and THV. With surgical aortic valve replacement, native annular calcifications are typically resected before valve implantation, whereas for transcatheter implantation, a certain amount and distribution is needed for prosthesis positioning and deployment. While annular calcifications have been reported to impact TAVI stent deployment and eccentricity [2,3], this is not likely impacting deformation of bio-sAVRs [23-25]. Deformation of bio-sAVRs might, however, be induced by the implantation technique, as well as by the annulus geometry that may change after annular calcification resection. It seems plausible that bio-sAVRs can undergo deformation due to the forces exerted by surgical sutures on the sewing ring, especially if the sutures are distributed unequally.

Deformation may also be related to the design of specific bio-sAVR types. Statistical analysis stratified for the degree of deformation by bio-sAVR type was not feasible in this study as valve type was dominated by CE Perimount (79\%), so results may be limited in regard to extrapolation across valve types.

We previously reported on the variation in change of the native aortic annulus throughout the cardiac cycle which has been shown to be predominantly oval in diastole and circular in systole [26, 27]. In our study, the bio-sAVRs showed a significantly lower EI (i.e., more circular shape) in diastole (5.1\% [IQR 3.0-7.6]) compared with systole (6.5\% [IQR 3.4-10.2]) suggesting that similar to the native annulus conformational changes occur with bio-sAVR albeit more circular in diastole for bio-sAVR vs. systole for native aortic valves.

Our study found a significantly higher deformity rate in bio-sAVRs with (suspected) valve pathology as compared with those imaged for routine or study purposes (median EI $6.9 \%$ versus $5.0 \%$, respectively), although the difference was relatively small. One could postulate that geometric deformity may lead to increase in leaflet malformation and thrombus formation similar to that of THV, but this is only hypothesis generating.

There are several limitations to our study. First, no interand intra-observer variability for EI measurements was performed. However, excellent reproducibility for diameter measurements in prosthetic valves using CT was shown previously using similar methodology [28]. Also, in THVs, no difference between MDCT and three-dimensional transesophageal echocardiography was found for annular eccentricity assessment [29]. Second, the time range from implant to MDCT was relatively large, with $34 \%$ performed $\leq 3$ months and $28.5 \%$ performed $\geq 5$ years after implantation. Third, because of the retrospective design, the impact of surgical implantation techniques could not be evaluated and the cohort was too small to study the effects of different bio-sAVR types. Future studies may provide insight on differences between specific sAVR types, implantation techniques, and the degree of deformation.

To conclude, biological sAVRs in this study showed mild geometric deformation in $39 \%$ of cases and were considered non-circular in an additional $17 \%$ of studied valves on MDCT at a median of 12 months (IQR 2-82) after implantation. We found a significantly higher deformity in aortic valves referred for imaging with suspected valve pathology compared with 
those being imaged for routine surveillance. Our results are hypothesis-generating and future studies are needed to evaluate the prognostic value of biological aortic valve deformation on structural valve durability and assess differences among valve types and surgical implantation techniques.

Funding information The authors state that this work has not received any funding.

\section{Compliance with ethical standards}

Guarantor The scientific guarantor of this publication is Ricardo Budde, Erasmus MC, Rotterdam.

Conflict of interest The authors of this manuscript declare no relationships with any companies, whose products or services may be related to the subject matter of the article.

Statistics and biometry No complex statistical methods were necessary for this paper.

Ethical approval Institutional Review Board approval was not required because the acquisition was a part of the routine clinical workup, and data were gathered retrospectively. No additional acquisitions were made specifically for this study. Patient data were retrieved from the electronic patient file.

\section{Methodology \\ - retrospective \\ - observational \\ - performed at three institutions}

\section{References}

1. Brown JM, O'Brien SM, Wu C, Sikora JA, Griffith BP, Gammie JS (2009) Isolated aortic valve replacement in North America comprising 108,687 patients in 10 years: changes in risks, valve types, and outcomes in the Society of Thoracic Surgeons National Database. J Thorac Cardiovasc Surg 137:82-90

2. Samad Z, Vora AN, Dunning A et al (2016) Aortic valve surgery and survival in patients with moderate or severe aortic stenosis and left ventricular dysfunction. Eur Heart J 37:2276-2286

3. Côté N, Pibarot P, Clavel MA (2017) Incidence, risk factors, clinical impact, and management of bioprosthesis structural valve degeneration. Curr Opin Cardiol 32:123-129

4. Hoffmann G, Lutter G, Cremer J (2008) Durability of bioprosthetic cardiac valves. Dtsch Arztebl Int 105:143-148

5. Binder RK, Webb JG, Toggweiler S et al (2013) Impact of postimplant Sapien XT geometry and position on conduction disturbances, hemodynamic performance, and paravalvular regurgitation. JACC Cardiovasc Interv 6:462-468

6. Delgado V, Ng AC, van de Veire NR et al (2010) Transcatheter aortic valve implantation: role of multi-detector row computed tomography to evaluate prosthesis positioning and deployment in relation to valve function. Eur Heart J 31:1114-1123

7. Willson AB, Webb JG, Gurvitch R et al (2012) Structural integrity of balloon-expandable stents after transcatheter aortic valve replacement: assessment by multidetector computed tomography. JACC Cardiovasc Interv 5:525-532
8. Fuchs A, De Backer O, Brooks M et al (2017) Subclinical leaflet thickening and stent frame geometry in self-expanding transcatheter heart valves. EuroIntervention 13:1067-1075

9. Caudron J, Fares J, Hauville C et al (2011) Evaluation of multislice computed tomography early after transcatheter aortic valve implantation with the Edwards Sapien bioprosthesis. Am J Cardiol 108: 873-881

10. Schultz CJ, Weustink A, Piazza N et al (2009) Geometry and degree of apposition of the corevalve revalving system with multislice computed tomography after implantation in patients with aortic stenosis. J Am Coll Cardiol 54:911-918

11. Wood DA, Tops LF, Mayo JR et al (2009) Role of multislice computed tomography in transcatheter aortic valve replacement. Am J Cardiol 103:1295-1301

12. Abbasi M, Azadani AN (2015) Leaflet stress and strain distributions following incomplete transcatheter aortic valve expansion. J Biomech 48:3663-3671

13. Rodríguez-Olivares R, Rahhab Z, El Faquir N et al (2016) Differences in frame geometry between balloon-expandable and self-expanding transcatheter heart valves and association with aortic regurgitation. Rev Esp Cardiol (Engl Ed) 69:392-400

14. Schoen FJ, Levy RJ (2005) Calcification of tissue heart valve substitutes: progress toward understanding and prevention. Ann Thorac Surg 79:1072-1080

15. Thubrikar MJ, Deck JD, Aouad J, Nolan SP (1983) Role of mechanical stress in calcification of aortic bioprosthetic valves. J Thorac Cardiovasc Surg 86:115-125

16. Sritharan D, Fathi P, Weaver JD, Retta SM, Wu C, Duraiswamy N (2018) Impact of clinically relevant elliptical deformations on the damage patterns of sagging and stretched leaflets in a bioprosthetic heart valve. Cardiovasc Eng Technol 9:351-364

17. Bernacca GM, Fisher AC, Wilkinson R, Mackay TG, Wheatley DJ (1992) Calcification and stress distribution in bovine pericardial heart valves. J Biomed Mater Res 26:959-966

18. Smith DB, Sacks MS, Pattany PM, Schroeder R (1999) Fatigueinduced changes in bioprosthetic heart valve three-dimensional geometry and the relation to tissue damage. J Heart Valve Dis 8:25-33

19. van Nooten G, Ozaki S, Herijgers P, Segers P, Verdonck P, Flameng W (1999) Distortion of the stentless porcine valve induces accelerated leaflet fibrosis and calcification in juvenile sheep. J Heart Valve Dis 8:34-41

20. Binder RK, Rodes-Cabau J, Wood DA et al (2013) Transcatheter aortic valve replacement with the Sapien 3: a new balloonexpandable transcatheter heart valve. JACC Cardiovasc Interv 6: 293-300

21. Blanke P, Reinohl J, Schlensak C et al (2012) Prosthesis oversizing in balloon-expandable transcatheter aortic valve implantation is associated with contained rupture of the aortic root. Circ Cardiovasc Interv 5:540-548

22. Willson AB, Rodes-Cabau J, Wood DA et al (2012) Transcatheter aortic valve replacement with the St. Jude medical portico valve: first-in-human experience. J Am Coll Cardiol 60:581-586

23. Haziza F, Papouin G, Barratt-Boyes B, Christie G, Whitlock R (1996) Tears in bioprosthetic heart valve leaflets without calcific degeneration. J Heart Valve Dis 5:35-39

24. Vesely I, Barber JE, Ratliff NB (2001) Tissue damage and calcification may be independent mechanisms of bioprosthetic heart valve failure. J Heart Valve Dis 10:471-477

25. Schoen FJ, Levy RJ (1999) Founder's Award, 25th Annual Meeting of the Society for Biomaterials, perspectives. Providence, RI, April 28-May 2, 1999. Tissue heart valves: current challenges and future research perspectives. J Biomed Mater Res 47:439-465

26. de Heer LM, Budde RP, van Prehn J et al (2012) Pulsatile distention of the nondiseased and stenotic aortic valve annulus: analysis with electrocardiogram-gated computed tomography. Ann Thorac Surg 93:516-522 
27. Sucha D, Tuncay V, Prakken NH et al (2015) Does the aortic annulus undergo conformational change throughout the cardiac cycle? A systematic review. Eur Heart J Cardiovasc Imaging 16:13071317

28. Suchá D, Daans CG, Symersky P et al (2015) Reliability, agreement, and presentation of a reference standard for assessing implanted heart valve sizes by multidetector-row computed tomography. Am J Cardiol 116:112-120
29. Ac N, Delgado V, van der Kley F et al (2010) Comparison of aortic root dimensions and geometries before and after transcatheter aortic valve implantation by 2 - and 3 -dimensional transesophageal echocardiography and multislice computed tomography. Circ Cardiovasc Imaging 3:94-102

Publisher's note Springer Nature remains neutral with regard to jurisdictional claims in published maps and institutional affiliations. 